\title{
Order parameter and current-phase relation in Josephson junctions composed of $g+s$-wave superconductors
}

\author{
Zengxu Wang, ${ }^{1}$ Weiyi Zhang, ${ }^{1}$ and Zidan Wang ${ }^{2}$ \\ ${ }^{1}$ Jiangsu Provincial Laboratory for NanoTechnology and Department of Physics, Nanjing University, Nanjing 210093, China \\ ${ }^{2}$ Department of Physics, The University of Hong Kong, Pokfulam Road, Hong Kong, China
}

(Received 26 January 2003; revised manuscript received 20 May 2003; published 19 November 2003)

\begin{abstract}
Based on the analyses of bulk sensitive experimental data on penetration depths, Raman spectra, electron photoemission spectra, etc., G. M. Zhao concluded in a recent paper [Phys. Rev. B 64, 024503 (2001)] that the symmetry of high- $T_{c}$ superconductors belongs to the $g+s$-wave type. To explore the common and uncommon features of the $g+s$-wave pairing state with respect to the $d$-wave pairing state, both superconductor-insulatorsuperconductor junction and superconductor-normal metal-superconductor junction have been studied selfconsistently in this paper using the quasiclassical theory. The current phase relation for $g+s$-wave superconductors $\Delta(s, \theta)=\Delta_{0}(s+\cos 4 \theta)$ is investigated systematically as functions of $s$-wave component, crystal orientation angle $\beta$, as well as roughness $\rho$ of the interface layer. Our results show that there exists a critical $\beta_{C}$ for a given $s$ and $\rho$ so that the current phase relation approaches asymptotically to $I(\varphi)=I_{C} \sin (2 \varphi)$ from $I(\varphi)=I_{C} \sin (\varphi)$ as $\beta \rightarrow \beta_{C}$. The order parameter and $\beta_{C}-S$ relation are calculated self-consistently as interface roughness varies. Our results are compared with their counterparts in Josephson junctions with the $d$-wave pairing state obtained using a similar method.
\end{abstract}

DOI: 10.1103/PhysRevB.68.184516

PACS number(s): 74.20.Rp, 74.50.+r

\section{INTRODUCTION}

The superconducting state is a macroscopic phase coherent state which is characterized by an order parameter; the determination of the order parameter symmetry is of vital importance for clarifying the superconducting mechanism. Since the discovery of high- $T_{c}$ superconductors in $1986,{ }^{1,2}$ a large amount of experimental data have been accumulated and analyzed which greatly improved our understanding on properties of both normal as well as superconducting states. A predominant $d$-wave pairing symmetry has been demonstrated through the scanning superconducting quantum interference device (SQUID) microscope in the phase sensitive tricrystals $^{3-6}$ and the Fraunhofer diffraction pattern of corner junctions between $s$-wave and high- $T_{c}$ superconductors. ${ }^{7,8}$ Direct measurement of the current phase relation (CPR) has also been carried out by Il'ichev and co-workers ${ }^{9-11}$ for the in-plane junctions between two high- $T_{c}$ superconductors, significant $\pi$ periodicity was observed at the crystal orientation angle $\beta_{C}=45^{\circ}$. However, $c$-axis Josephson junctions involving both conventional superconductor $(\mathrm{Pb})$ and high$T_{c}$ superconductors ${ }^{12-14}$ consistently show that Shapiro steps take place only at the multiples of $V=h f / 2 e$. These results indicate that a sizeable $s$-wave component exists in high- $T_{c}$ superconductors. Meanwhile, comprehensive theoretical investigations reveal that spin fluctuation near antiferromagnetic instability plays an important role. ${ }^{15,16}$ Not only can many anomalous properties in the normal state be explained within the spin-fluctuation mechanism, it also predicts the $d$-wave pairing state as the candidate for the superconducting state. The symmetry of the pairing states also has direct manifestation on the Josephson effect; the CPR relation takes approximately the $\sin 2 \varphi$ pattern if a Josephson junction is made between $s$-wave and $d$-wave superconductors along the $c$ axis. ${ }^{17-19}$ The same behavior was shown also for the in- plane junction between two $d$-wave superconductors rotated with each other by $45^{\circ} .{ }^{20-22}$ One of the interesting features predicted theoretically ${ }^{23}$ for the anisotropic $d$-wave pairing state is the zero-energy state. It results from the phase change along the quasiparticle trajectory in the vicinity of surfaces or interfaces and accompanied depletion of the order parameter; the well structures of order parameter enables the formation of the bound state near the Fermi energy. The zeroenergy bound states ${ }^{23-25}$ can greatly enhance the critical current at low temperature and induces a sign change in the critical current.

The experimental techniques in measuring the order parameter symmetry can be generally classified into two types, i.e., the bulk sensitive and surface sensitive probes. Unlike the conventional isotropic superconductors, where surface does not play a significant role, the electronic structures in anisotropic superconductors can be very different from the bulk and surface pair breaking effect can be dramatic. Thus the bulk sensitive and surface sensitive measurements can some times reveal contradictory conclusion concerning the paring state symmetry. To reconcile such discrepancy, several proposals have been made recently regarding the Josephson effect in junctions involving high- $T_{c}$ superconductors. Kuboki and Sigrist ${ }^{26}$ and Sigrist ${ }^{27}$ found that a $s$-wave component arises if local time-reversal symmetry is broken near interface in $d$-wave superconductors, but the effect vanishes when the relative $a$-axis's angle approaches $45^{\circ}$. A similar Ginzburg-Landau formalism by Ren, Xu, and Ting ${ }^{28,29}$ found that a small $s$-wave component near a surface is always locked in phase with $d$-wave component to form a real combination. The existence of the broken time-reversal symmetry states requires a phase change in the order parameter along the quasiparticle trajectory; ${ }^{30,31}$ it may happen solely for the in-plane junction and not for the $c$-axis junction. However, the broken time-reversal symmetry state was not 
observed up to now in the scanning SQUID microscope in tricrystal samples. ${ }^{6}$ In addition, the usual Shapiro steps $V$ $=n h f / 2 e$ in $c$-axis Josephson junctions also invoked other proposals as well, such as the deformation of the Fermi surface in the $\mathrm{Pb},{ }^{32}$ transformation of $d$-wave pairing state into $s$-wave pairing state due to surface scattering, etc. ${ }^{33,34}$

Very recently, G. M. Zhao wrote a comprehensive paper ${ }^{35}$ on the identification of the order parameter symmetry in high- $T_{c}$ superconductors and proposed the $g+s$ wave as the most probable candidate. His conclusion is based on the analyses on a variety of experimental measurements on the bulk sensitive properties, such as thermal conductivity, angle-resolved photoemission (since coherent length is very small), Raman spectra, specific heat, penetration depth, and Knight shift, etc. The model calculations with $g+s$-wave order parameter $\Delta(s, \theta)=\Delta_{0}(s+\cos 4 \theta)$ not only gives excellent fittings to the above experimental data, but also gives an excellent explanation to the electronic tunneling spectra in single tunneling breaking junctions. The comparison with the experimental data yield $\Delta_{0}=17.75 \mathrm{meV}$ and $s=0.46$ for the slightly overdoped $\mathrm{Bi}_{2} \mathrm{Sr}_{2} \mathrm{CaCu}_{2} \mathrm{O}_{8+y} \quad\left(T_{c}=90 \mathrm{~K}\right),{ }^{35} \Delta_{0}$ $=24.5 \mathrm{meV}$ and $s=0.225$ for the slightly overdoped $\mathrm{YBa}_{2} \mathrm{Cu}_{3} \mathrm{O}_{7-x},{ }^{35}$ and $\Delta_{0}=8.1 \mathrm{meV}$ and $s=0.53$ for the nearly optimally doped $\mathrm{La}_{2-x} \mathrm{Sr}_{x} \mathrm{CuO}_{4}{ }^{35}$ The bulk sensitive measurement data are supposed to reflect the intrinsic nature of these high- $T_{c}$ superconductors and avoid the surface contamination or degradation which can appear in surface sensitive experiments. However, the important issue regarding to the explanation of the scanning SQUID microscope in phase sensitive tricrystals ${ }^{3-6}$ is not convincingly presented and is attributed to the possible surface effect. Though it is still an open question concerning the relevance of $g+s$-wave pairing state to the high- $T_{c}$ cuprates, $g+s$-wave symmetry was also recently proposed as a candidate in the borocarbide superconductor. ${ }^{36}$

To explore the possible surface impact on the order parameter as well as on the phase sensitive tricrystal measurement, ${ }^{3-6}$ the surface pair breaking effect has to be calculated self-consistently in order to check whether the order parameter at the surface is fundamentally different from the bulk and whether the order parameter forms a potential well structure for a certain quasiparticle trajectory. Thus we have studied in this paper the self-consistent order-parameter profile and the CPR relation for the in-plane Josephson junction between two $g+s$-wave superconductors. Although high- $T_{c}$ superconductors are strongly correlated electronic systems, ${ }^{15,16}$ we will concentrate on the region where high$T_{c}$ superconductors can be treated as a Fermi liquid. Overdoped and optimally doped high- $T_{c}$ superconductors belong to such a category. A very useful formulation of the Fermiliquid theory of superconductivity is based on the quasiclassical transport theory, ${ }^{37,38}$ it describes slowly varying phenomena in space and time with the requirements that order parameter $\Delta$ is much smaller than the Fermi energy $E_{F}$ and coherence length $\xi_{0}=\hbar v_{F} / 2 \pi k_{B} T_{c}$ is much larger than the Fermi wavelength $k_{F}^{-1} \cdot{ }^{39}$ For high- $T_{c}$ superconductors, $E_{F}$ $\sim 0.2 \mathrm{eV},{ }^{40,41} \Delta \sim 0.02 \mathrm{eV}, \Delta / E_{F} \sim 0.1$, and $1 /\left(\xi_{0} k_{F}\right) \sim 0.1$; they are still reasonably small although large in comparison with those of conventional superconductors. Since the quasiclassical theory is expanded in terms of these small param- eters, the conclusion drawn from these calculations should be qualitatively correct though it may be quantitatively in error by $10 \%$.

As demonstrated by Tsuei and Kirtley ${ }^{3-6}$ in the phase sensitive tricrystal measurements, a specially designed inclination angle $\theta=75^{\circ}$ can be used to distinguish the $d$-wave pairing state from the $g$-wave pairing state. For the $g+s$-wave pairing state, the diagrammatic analysis does not alter the conclusion for a moderate $s$-wave component. Thus, unless the surface scattering fundamentally changes the symmetry of the order parameter near surface, the zero frustrated phase at inclination angle $\theta=75^{\circ}$ can be used as a smoking gun to pin down the symmetry. To this aim, we have computed the order parameter and CPR relation self-consistently for a Josephson junction composed of $g+s$-wave superconductors in the presence of a rough interface. Our results show that interface scattering does not change the symmetry of the order parameter, but the ratio between the $g$-wave and $s$-wave components can alter because the anisotropic $g$-wave component is more vulnerable to the interface scattering than the $s$-wave component. The impact of interface roughness causes depletion of the order parameter near the interface and it also reduces the critical tunneling current. The numerical analysis also shows that there exists a critical $\beta_{C}$ for a given $s$ and $\rho$ so that the CPR relation approaches asymptotically to $I(\varphi)=I_{C} \sin (2 \varphi)$ from $I(\varphi)=I_{C} \sin (\varphi)$ as $\beta$ $\rightarrow \beta_{C}$. The order parameter and $\beta_{C^{-S}}$ relation are calculated self-consistently as interface roughness varies. Our results are compared with previous theoretical studies on Josephson junctions with $d$-wave superconductors. For a pure $d$-wave superconductor, it is well known theoretically that $I(\varphi)$ $=I_{c} \sin (2 \varphi)$ occurs at $\beta_{C}=\pi / 4$ for the in-plane junction, while for pure $g$-wave superconductors, the same behavior takes place at $\beta_{C}=\pi / 8$. For a general $g+s$-wave superconductor, $\beta_{C}$ increases with the $s$-wave component and approaches $\beta=\pi / 4$ if $s$ is large. The depletion of the order parameter results in the zero-energy bound state which can be calculated from the retarded Green's function using the above self-consistently determined order-parameter profile.

In Sec. II, we will first briefly describe the formulism of quasiclassical approach ${ }^{39}$ as it applies to Josephson problems. In Sec. III, both superconductor-insulatorsuperconductor (SIS) junction and superconductor-normalmetal-superconductor (SNS) junction are investigated, and detailed numerical results on the CPR relation are discussed and compared with those obtained for pure $d$-wave superconductors. The conclusion is given in Sec. IV.

\section{QUASICLASSICAL METHOD}

As we mentioned in the Introduction, the order parameter and current phase relation in the Josephson junction can be conveniently computed within the quasiclassical theory. For the equilibrium state, the superconducting state is described by the $2 \times 2$ Matsubara propagator $g^{M}\left(\hat{k}, \vec{R} ; \epsilon_{n}\right)$ in particlehole space, which satisfies the transportlike equation ${ }^{39}$

$$
\left[i \epsilon_{n} \hat{\tau}_{3}-\hat{\Delta}, \hat{g}^{M}\left(\hat{k}, \vec{R} ; \epsilon_{n}\right)\right]_{-}+i \hbar v_{F} \hat{k} \cdot \vec{\nabla}_{\vec{R}} \hat{g}^{M}\left(\hat{k}, \vec{R} ; \epsilon_{n}\right)=0
$$


and normalization condition

$$
\left[\hat{g}^{M}\left(\hat{k}, \vec{R} ; \epsilon_{n}\right)\right]^{2}=-(\pi \hbar)^{2} .
$$

$\hat{\Delta}$ and $\epsilon_{n}=k_{B} T(2 n+1)$ are the superconducting order parameter and the Matsubara frequency, $\hat{k}$ denotes the trajectory of the propagator, and $\hat{\tau}_{3}$ is the third Pauli matrix in particle-hole space. In the bulk superconductor, Eq. (1) forms a closed set together with the self-consistent equation for the order parameter,

$$
\begin{aligned}
\hat{\Delta}_{12}(\hat{k}, \vec{R})= & \frac{k_{B} T}{\hbar} \sum_{n}{ }^{\prime} \int \frac{d \Omega_{\hat{k}^{\prime}}}{4 \pi} f(\hat{k}) f\left(\hat{k}^{\prime}\right) \hat{g}_{12}^{M}\left(\hat{k}^{\prime}, \vec{R}, \epsilon_{n}\right) \\
& \times\left(\ln \left(T / T_{C}\right)+\sum_{n}{ }^{\prime} \frac{1}{2 n+1}\right)^{-1} .
\end{aligned}
$$

Here, the prime means a cut off on the frequency summation and the function $f(\hat{k})$ denotes the orbital wave function of the Cooper pair. The superconducting current can be calculated from

$$
\vec{J}=\frac{k_{B} T}{R_{0} e \hbar} \sum_{n}{ }^{\prime} \int \frac{d \Omega_{\hat{k}}}{4 \pi} \hat{k} \operatorname{Re} g^{M}\left(\hat{k}, \vec{R}, \epsilon_{n}\right),
$$

where $e$ is the electron charge and $R_{0}=\left[2 N\left(E_{F}\right) e^{2} v_{F}\right]^{-1}$ is the Sharvin resistance. ${ }^{42}$

The rough interface can be modeled by a strongly scattered impurity layer ${ }^{38,43}$ which can be formulated mathematically as

$$
\left[\hat{g}^{M}\left(\hat{k}, \xi ; \epsilon_{n}\right),\left\langle\hat{g}^{M}\right\rangle\left(\xi, \epsilon_{n}\right)\right]_{-}+\frac{2 \pi i}{\rho} \hbar k_{\perp} \frac{d}{d \xi} \hat{g}^{M}\left(\hat{k}, \xi ; \epsilon_{n}\right)=0
$$

with

$$
\left\langle\hat{g}^{M}\right\rangle\left(\xi, \epsilon_{n}\right)=\int \frac{d \Omega_{\hat{k}}}{4 \pi} \hat{g}^{M}\left(\hat{k}, \xi ; \epsilon_{n}\right)
$$

denoting the impurity self-energy. $k_{\perp}$ is the projection of trajectory along the interface normal, $\rho$ is the roughness parameter of the interface and is related to the conventional diffusivity parameter $p$ (Ref. 44) through the relation $p=1$ $-4 \int_{0}^{\pi / 2} d \theta \cos \theta \sin ^{3} \theta \exp (-\rho / \cos \theta)$ with $p(\rho=0)=0$ standing for the transparent interface and $p(\rho=\infty)=1$ for the fully diffuse interface. $\xi= \pm 1 / 2$ corresponds to $\vec{R}=\vec{R}_{\text {surf }}$ $\pm 0^{+}$, where $\vec{R}_{\text {surf }}$ is the coordinate of the interface layer.

In this paper, we consider the in-plane SIS and SNS junctions depicted in Fig. 1. The Cartesian coordinate is chosen such that the $y z$ plane is within the cross section of the junction and the $x$ axis is along the junction. Two identical $g$ $+s$-wave superconducting single crystals have their $c$ axes parallel to the $z$ axis. The crystal axes of the left superconductor is fixed with $a \| x$ and $b \| y\left(\alpha=0^{\circ}\right)$, while the right superconductor is allowed to rotate along the $z$ axis. We use $\beta$ to denote the angle between the crystal axis $a$ of the right superconductor and coordinate axis $x$. By studying the Josephson effect as a function of $\beta$, we seek to find out the common and uncommon features for the $g+s$-wave super-

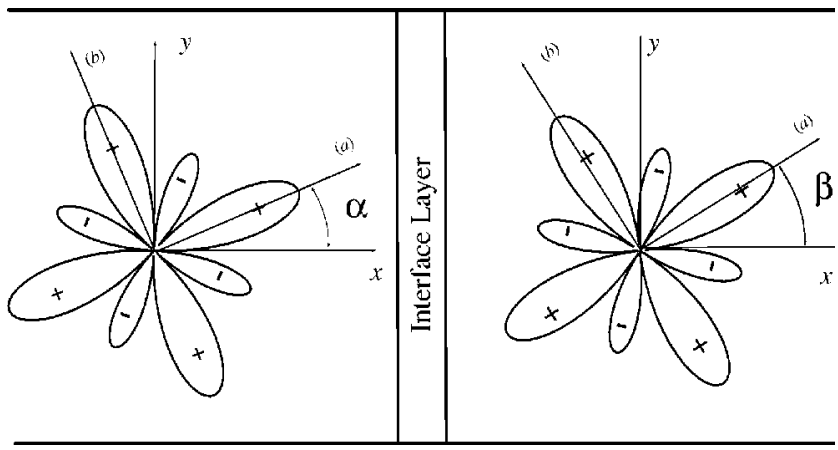

FIG. 1. The schematic diagram for the planar Josephson junction under consideration. $\alpha$ and $\beta$ refer to the angles between interface normal ( $x$ axis) and crystal $a$ axis ( $\mathrm{Cu}-\mathrm{Cu}$ bond) of left and right superconductors, respectively.

conductors. For simplicity, we assume a cylindrical Fermi surface for the high- $T_{c}$ superconductor, we also take the Fermi momentums and velocities in both superconductors and normal-metal layer as equal. The width of the normalmetal layer is set to $d=3.56 \xi_{0}\left(\xi_{0}=\hbar v_{F} / 2 \pi k_{B} T_{c}\right)$. For Josephson junction problems, the phase difference of the order parameter between the right and left bulk superconductors is fixed and the order parameter in the bulk is given by

$$
\hat{\Delta}_{12}(\hat{k}, x)= \begin{cases}\Delta(T) f(\hat{k}) \exp (\varphi / 2), & x \gg d / 2 ; \\ \Delta(T) f(\hat{k}) \exp (-\varphi / 2), & x \ll-d / 2 .\end{cases}
$$

$f(\hat{k})=1, \quad \sqrt{2}\left(\hat{k}_{a}^{2}-\hat{k}_{b}^{2}\right), \quad$ and $\quad \sqrt{2 /\left(1+2 s^{2}\right)}\left[s+\left(\hat{k}_{a}^{4}+\hat{k}_{b}^{4}\right.\right.$ $\left.\left.-6 \hat{k}_{a}^{2} \hat{k}_{b}^{2}\right)\right]$ correspond to the conventional $s$-wave,

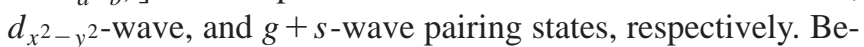
low we will concentrate on the Josephson effect for the $g+$ $s$-wave superconductor.

\section{RESULTS AND DISCUSSIONS}

For the numerical calculation, we choose the reduced temperature $T=0.4 T_{c}$. The above set of equations are solved self-consistently using the iteration scheme for both superconductor-insulator-superconductor (SIS) and superconductor-normal-metal-superconductor (SNS) junctions, the results are presented below separately for the two cases.

\section{A. Superconductor-insulator-superconductor junction}

For the SIS Josephson junction, the insulator layer is mimicked with a strongly scattered impurity layer and barrier height is modeled by a roughness parameter $\rho$. Two values for the roughness parameter $\rho=1.27$ and $\rho=2.16$ are considered. Our result suggests that the current-phase relation for the $g+s$-wave superconductor depends sensitively on the interplay among the $s$-wave component, roughness of the insulator layer, and the crystal orientation of the right superconductors. For the pure $g$-wave superconductor, $\beta_{C}$ $=22.5^{\circ}$ corresponds to the configuration with maximum order parameter on the left and the minimum order parameter on the right of the junction, thus the second-order terms van- 


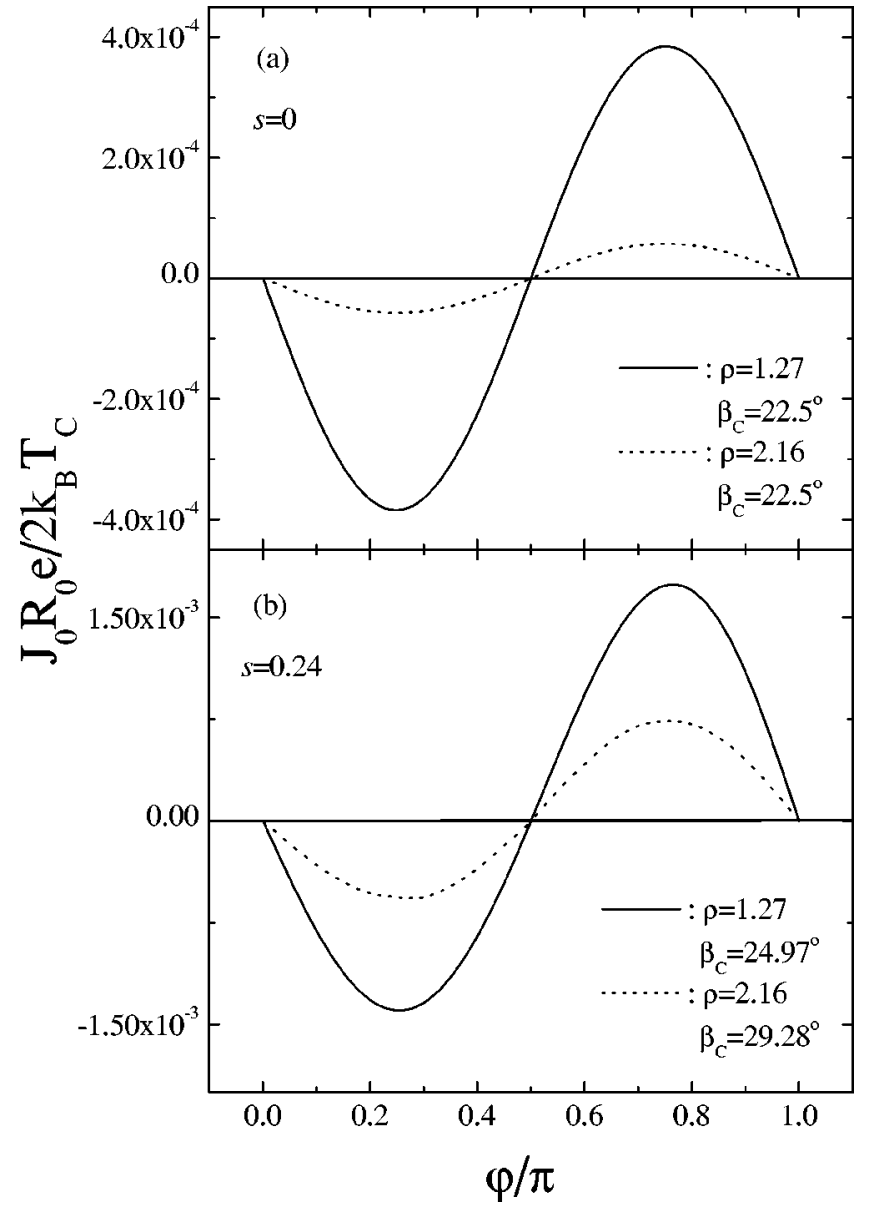

FIG. 2. The CPR relation in SIS junctions at $\beta_{C}$. (a) $s=0$; (b) $s=0.24$. The solid and dotted lines correspond to $\rho=1.27$ and $\rho$ $=2.16$, respectively.

ish, but the fourth-order terms survive, and the current-phase relation takes the form $I(\varphi)=I_{c} \sin (2 \varphi)$ as shown in Fig. 2(a). The roughness parameter $\rho$ does reduce the magnitude of the critical superconducting current, but the $\pi$ periodicity is not altered. Once a realistic $g+s$-wave order parameter $\Delta=\Delta_{0}(s+\cos 4 \theta)(s=0.24)$ is taken into account, the $\pi$ periodicity does still occur, but the critical $\beta_{C}$ is a sensitive function of roughness parameter since $s$-wave and $g$-wave components respond to roughness in a very different way as we mentioned in the Introduction. Illustrated in Fig. 2(b) is the CPR relation for $s=0.24$; the critical angle is $\beta_{C}$ $=24.97^{\circ}$ for $\rho=1.27$ and $\beta_{C}=29.28^{\circ}$ for $\rho=2.16$, respectively. In addition, similar mirror configuration exists for the $\pi$ periodicity at $90^{\circ}-\beta_{C}$. The experimental proof for $\pi$ periodicity is to check whether the Shapiro step appears at $V=h \nu / 4 e$ or the Fraunhofer diffraction pattern takes the form $I_{\text {max }}(\Phi)=I_{c}\left|\sin \left(2 \pi \Phi / \Phi_{0}\right) /\left(2 \pi \Phi / \Phi_{0}\right)\right|$. However, $\pi$ periodicity for the $g+s$-wave order parameter is not only a symmetry issue, but also depends on the detailed sample preparation; it is even more difficult to be observed.

The evolution of the Josephson junction from $2 \pi$ periodicity to $\pi$ periodicity in the neighborhood of $\beta_{C}$ is plotted in Fig. 3; it is seen that $\beta_{C}$ is a crossover point which divides the 0 junction and $\pi$ junction. The $\beta$ range for significant $\pi$

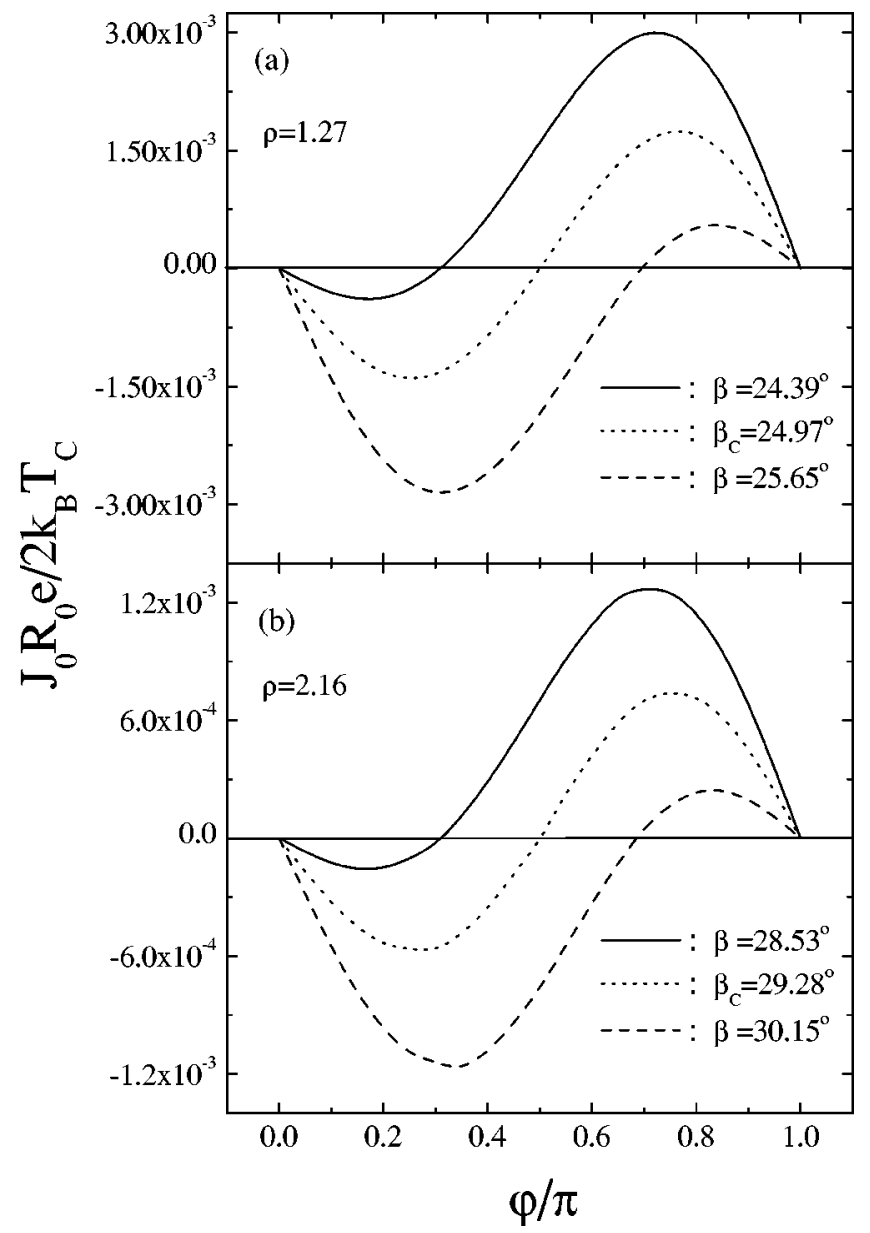

FIG. 3. The CPR relation in SIS junctions in the vicinity of $\beta_{C}$ at $s=0.24$. (a) $\rho=1.27$. The solid, dotted, and dashed lines correspond to $\beta=24.39^{\circ}, \beta_{C}=24.97^{\circ}$, and $\beta=25.65^{\circ}$, respectively; (b) $\rho=2.16$. The solid, dotted, and dashed lines correspond to $\beta$ $=28.53^{\circ}, \beta_{C}=29.28^{\circ}$, and $\beta=30.15^{\circ}$, respectively.

periodicity is rather narrow: $\Delta \beta=1.26^{\circ}$ when the roughness parameter $\rho=1.27$ and $\Delta \beta=1.62^{\circ}$ when $\rho=2.16$. A large surface roughness reduces the $g$-wave component more strongly and makes the $g$-wave and $s$-wave components

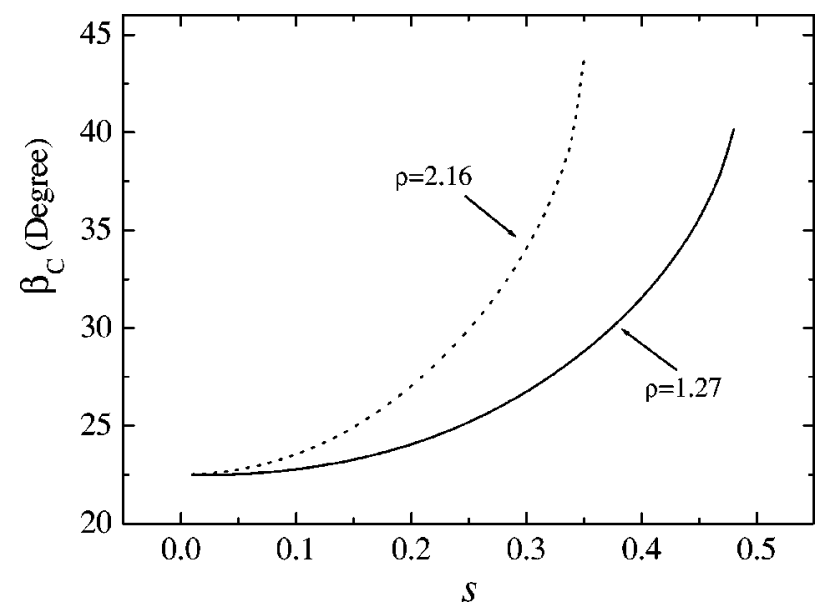

FIG. 4. The $\beta_{C}$ versus $s$ in SIS Josephson junction. The solid and dotted lines correspond to $\rho=1.27$ and $\rho=2.16$, respectively. 


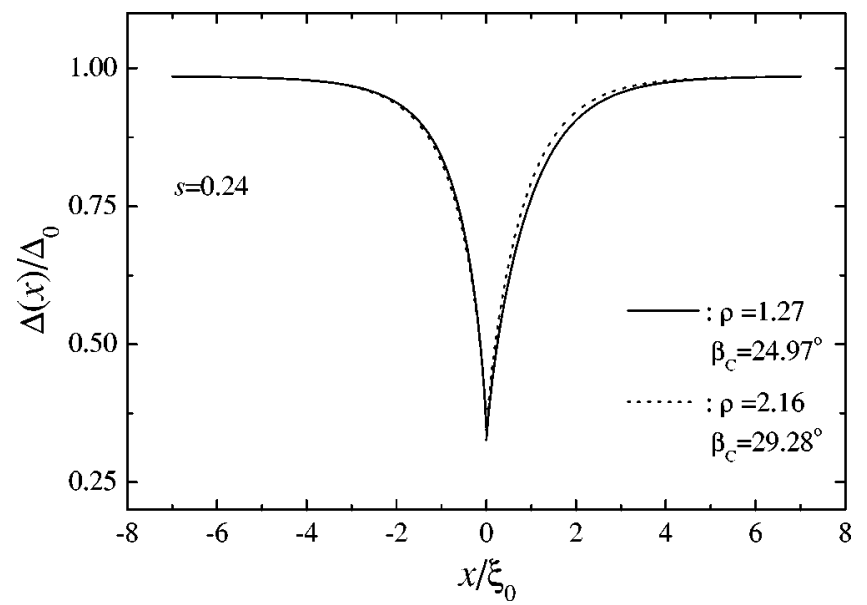

FIG. 5. The order-parameter profiles in SIS junctions at $s$ $=0.24$. The solid and dotted lines refer to $\rho=1.27, \beta_{C}=24.97^{\circ}$ and $\rho=2.16, \beta_{C}=29.28^{\circ}$, respectively.

more compatible. In Fig. 4 , the $\beta_{C}$ is shown as a function of $s$ for two sets of roughness parameters. It is clear that $\beta_{C}$ is a monotonic function of both $s$ and $\rho ; \beta_{C}$ increases from $22.5^{\circ}$ to a angle approaching to $45^{\circ}$ as $s$ increases. Thus the observation of significant fourth-order terms near $\beta=45^{\circ}$ is not only consistent with the dominant $d$-wave scenario, but

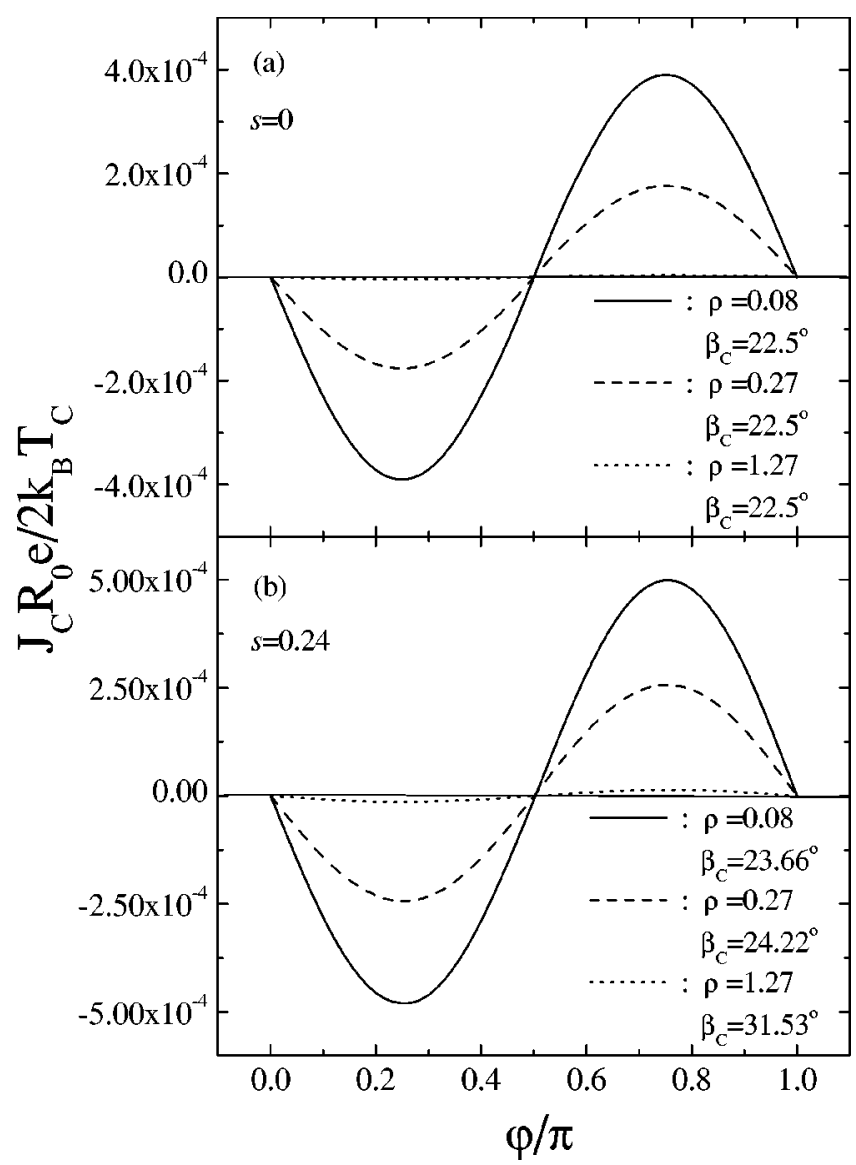

FIG. 6. The CPR relation in SNS junctions at $\beta_{C}$. (a) $s=0$; (b) $s=0.24$. The solid, dotted, and dashed lines corresponds to $\rho$ $=0.08, \rho=0.27$, and $\rho=1.27$, respectively.

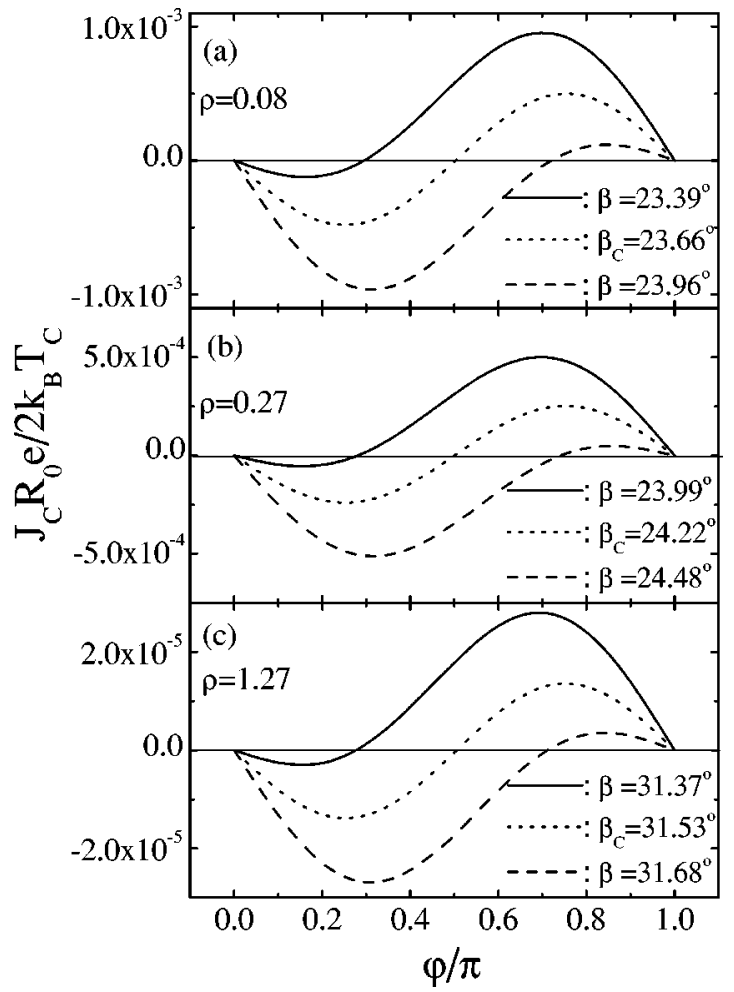

FIG. 7. The CPR relation in SNS junctions in the vicinity of $\beta_{C}$ at $s=0.24$. (a) $\rho=0.08$, the solid, dotted, and dashed lines correspond to $\beta=23.39^{\circ}, \beta_{C}=23.66^{\circ}$, and $\beta=23.96^{\circ}$; (b) $\rho=0.27$, the solid, dotted, dashed lines correspond to $\beta=23.99^{\circ}, \beta_{C}=24.22^{\circ}$, and $\beta=24.48^{\circ}$; (c) $\rho=1.27$, the solid, dotted, dashed lines correspond to $\beta=31.37^{\circ}, \beta_{C}=31.53^{\circ}$, and $\beta=31.68^{\circ}$.

also consistent with the $g+s$-wave scenario when the $s$-wave component is large. Finally, the order-parameter profiles near the interface are demonstrated in Fig. 5. The rough insulator has strong pair breaking effect on the $g$-wave component, but has weak influence on the $s$-wave component. The orderparameter profile shows a pronounced dip in the vicinity of the interface, such dip is responsible for the zero-energy bound state. Since the order-parameter profile is calculated self-consistently in our study, the contribution of the zeroenergy bound state as well as other bound states are implicitly included in our tunneling current. ${ }^{45}$

\section{B. Superconductor-normal-metal-superconductor junction}

In carrying out the similar calculations on SNS Josephson junction, we also assume that the interface between the normal metal and superconductors can be rough. To simplify the calculation, the roughness of the two interfaces is taken to be the same and values of $\rho=0.08,0.27,1.27$ are considered. For the pure $g$-wave superconductor, the $\pi$ periodicity occurs at the same crystal orientation angle $\beta_{C}=22.5^{\circ}$ and is independent of $\rho$; this is shown in Fig. 6(a). The results resemble that of the SIS junction and are determined solely by the symmetry of $g$-wave order parameter, but the overall magnitude for the same roughness parameter is very much reduced. For a realistic $g+s$-wave pairing state with $s=0.24$, the CPR relations at critical $\beta_{C}$ are given in Fig. 6(b). The $\beta_{C}$ 


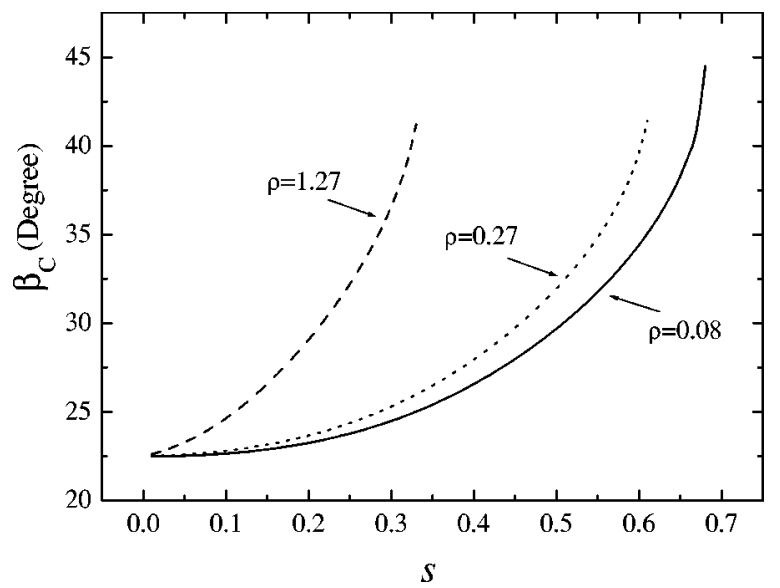

FIG. 8. The $\beta_{C}$ versus $s$ in SNS Josephson junction. The solid, dotted, and dashed lines correspond to $\rho=0.08, \rho=0.27$, and $\rho$ $=1.27$, respectively.

depends sensitively on roughness $\rho, \beta_{C}=23.66^{\circ}$ for $\rho$ $=0.08 ; \beta_{C}=24.22^{\circ}$ for $\rho=0.27$, and $\beta_{C}=31.53^{\circ}$ for $\rho$ $=1.27$, respectively.

In the vicinity of critical crystal orientation angle $\beta_{C}$, the CPR relation changes continuously from $2 \pi$ periodicity to $\pi$ periodicity and the angular range for this transition is even narrower. The typical CPR relations in this transition range are plotted in Fig. 7 for three sets of roughness parameters and three sets of crystal orientation angles. The significant $\pi$ periodicity can be observed within $\Delta \beta=0.57^{\circ}$ for $\rho=0.08$, $\Delta \beta=0.49^{\circ}$ for $\rho=0.27$, and $\Delta \beta=0.31^{\circ}$ for $\rho=1.27$, respectively. Comparing with those of the SIS junction, $\Delta \beta$ in the SNS junction is much smaller. The dependence of $\beta_{C}$ with $s$ is shown in Fig. 8; $\beta_{C}$ is also a monotonic function of $s$ and approaches $45^{\circ}$ when the $s$-wave component is compatible with the $g$-wave component. The self-consistent order-parameter profile in the SNS junction is plotted in Fig. 9; the overall feature is quite similar to that of the SIS junction.

From the above results, we found that the rough interface can suppress the order parameter in its vicinity, but the symmetry of the order parameter is maintained throughout the region if no new pairing channel is introduced near the interface. In this sense, the phase sensitive tricrystal measurement at inclination angle $\theta=75^{\circ}$ cannot be explained by the $g+s$-wave pairing state in the presence of surface scattering. Also the self-consistently determined order parameter suggests that zero-energy bound states are always possible for a certain quasiparticle trajectory due to a potential well created by a pair breaking effect; such effect causes a sign change in the critical current at low temperature since the contribution of the zero-energy state is then greatly enhanced.

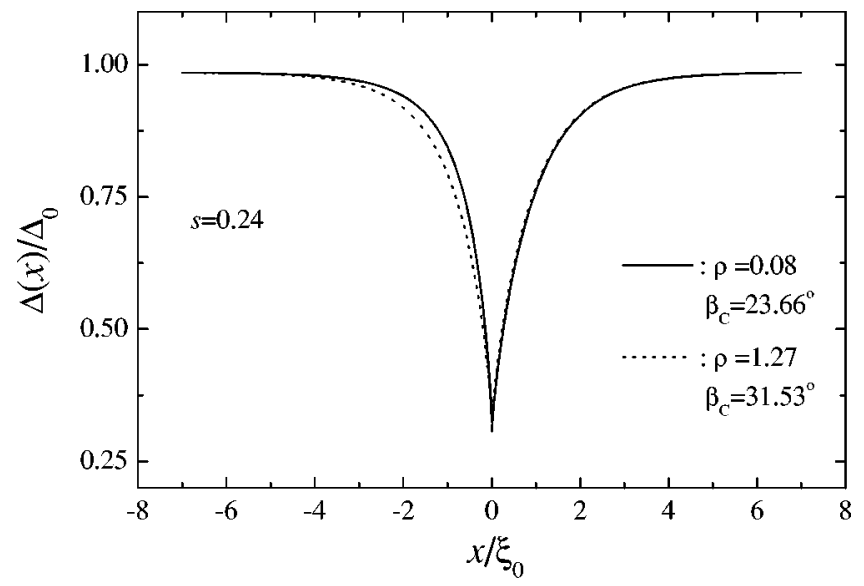

FIG. 9. The order-parameter profiles in SNS junctions at $s$ $=0.24$. The solid and dotted lines refer to $\rho=0.08, \beta_{C}=23.66^{\circ}$ and $\rho=1.27, \beta_{C}=31.53^{\circ}$, respectively.

\section{CONCLUSION}

In summary, we have studied in this paper the Josephson effect of the planar junction consisting of $g+s$-wave superconductors. For the pure $g$-wave pairing state, the $\pi$ periodicity can take place at the crystal orientation angle $\beta_{C}$ $=22.5^{\circ}$. This property is solely determined by the pairing symmetry of the $g$-wave order parameter. While for the general $g+s$-wave pairing state, $\pi$ periodicity can also appear under the suitable combination among the $s$-wave component, interface roughness, and crystal orientation angle, but the angular range for $\pi$ periodicity is rather narrow and makes the observation technically quite difficult. An interesting point of our calculation is that the critical orientation angle $\beta_{C}$ approaches $45^{\circ}$ if the $s$-wave component is compatible with the $g$-wave component. Thus the significant fourth-order terms in CPR measurement observed by Il'ichev and co-workers ${ }^{9-11}$ is not only consistent with the $d$-wave, but also consistent with $g+s$-wave pairing state. However, our study also shows that surface scattering does not change the symmetry of the order parameter in the surface region, thus it is unable to account for the zero frustrated phase observed in the phase sensitive tricrystal at inclination angle $\theta=75^{\circ}$.

\section{ACKNOWLEDGMENT}

The present work was supported in part by the National Natural Science Foundation of China under Grant Nos. 19934003, 90103022, the "Excellent Youth Foundation" Grant No. 10025419, the "Climbing Program" of NSTC (Grant No. G19980614), and the RGC grant of Hong Kong under Grant No. HKU7092/01P.
${ }^{1}$ J.G. Bednorz and K.A. Müller, Z. Phys. B: Condens. Matter 64, 189 (1986)

${ }^{2}$ M.K. Wu, J.R. Ashburn, C.J. Torng, P.H. Hor, R.L. Meng, L. Gao, Z.J. Huang, Y.Q. Wang, and C.W. Chu, Phys. Rev. Lett. 58, 908
(1987).

${ }^{3}$ C.C. Tsuei, J.R. Kirtley, Z.F. Ren, J.H. Wang, H. Raffy, and Z.Z. Li, Nature (London) 387, 481 (1997).

${ }^{4}$ J.R. Kirtley, C.C. Tsuei, and K.A. Moler, Science 285, 1373 
(1999).

${ }^{5}$ C.C. Tsuei and J.R. Kirtley, Phys. Rev. Lett. 85, 182 (2000).

${ }^{6}$ C.C. Tsuei and J.R. Kirtley, Rev. Mod. Phys. 72, 969 (2001), and references therein.

${ }^{7}$ D.A. Wollman, D.J. Van Harlingen, W.C. Lee, D.M. Ginsberg, and A.J. Leggett, Phys. Rev. Lett. 71, 2134 (1993).

${ }^{8}$ K.A. Kouznetsov, A.G. Sun, B. Chen, A.S. Katz, S.R. Bahcall, J. Clarke, R.C. Dynes, D.A. Gajewski, S.H. Han, M.B. Maple, J. Giapintzakis, J.-T. Kim, and D.M. Ginsberg, Phys. Rev. Lett. 79, 3050 (1997).

${ }^{9}$ E. Il'ichev, V. Zakosarenko, R.P.J. IJsselsteijn, V. Schultze, H.-G. Meyer, H.E. Hoenig, H. Hilgenkamp, and J. Mannhart, Phys. Rev. Lett. 81, 894 (1998).

${ }^{10}$ E. Il'ichev, V. Zakosarenko, R.P.J. IJsselsteijn, H.E. Hoenig, V. Schultze, H.-G. Meyer, M. Grajcar, and R. Hlubina, Phys. Rev. B 60, 3096 (1999).

${ }^{11}$ E. Il'ichev, M. Grajcar, R. Hlubina, R.P.J. IJsselsteijn, H.E. Hoenig, H.-G. Meyer, A. Golubov, M.H.S. Amin, A.M. Zagoskin, A.N. Omelyanchouk, and M.Yu. Kupriyanov, Phys. Rev. Lett. 86, 5369 (2001).

${ }^{12}$ R. Kleiner, A.S. Katz, A.G. Sun, R. Summer, D.A. Gajewski, S.H. Han, S.I. Woods, E. Dantsker, B. Chen, K. Char, M.B. Maple, R.C. Dynes, and J. Clarke, Phys. Rev. Lett. 76, 2161 (1996).

${ }^{13}$ M. Mößle and R. Kleiner, Phys. Rev. B 59, 4486 (1999).

${ }^{14}$ Q. Li, Y.N. Tsay, M. Suenaga, R.A. Klemm, G.D. Gu, and N. Koshizuka, Phys. Rev. Lett. 83, 4160 (1999).

${ }^{15}$ D.J. Scalapino, Phys. Rep. 250, 329 (1995).

${ }^{16}$ Toru Moriya and Kazuo Ueda, Adv. Phys. 49, 555 (2000).

${ }^{17}$ Y. Tanaka, Phys. Rev. Lett. 72, 3871 (1994).

${ }^{18}$ Y. Tanaka and S. Kashiwaya, Phys. Rev. B 56, 892 (1997).

${ }^{19}$ S. Kashiwaya and Yukio Tanaka, Rep. Prog. Phys. 63, 1641 (2000).

${ }^{20}$ S.K. Yip and J.A. Sauls, Phys. Rev. Lett. 69, 2264 (1992).

${ }^{21}$ S.K. Yip, Phys. Rev. B 52, 3087 (1995).

${ }^{22}$ W. Zhang, Phys. Rev. B 52, 3772 (1995); 52, 12538 (1995).

${ }^{23}$ Chia-Ren Hu, Phys. Rev. Lett. 72, 1526 (1994).
${ }^{24}$ Yukio Tanaka and Satoshi Kashiwaya, Phys. Rev. Lett. 74, 3451 (1995).

${ }^{25}$ Yukio Tanaka and Satoshi Kashiwaya, Phys. Rev. B 53, R11 957 (1996).

${ }^{26}$ K. Kuboki and M. Sigrist, J. Phys. Soc. Jpn. 65, 361 (1996).

${ }^{27}$ M. Sigrist, Prog. Theor. Phys. 99, 899 (1998).

${ }^{28}$ Y. Ren, Ji-Hai Xu, and C.S. Ting, Phys. Rev. Lett. 74, 3680 (1995).

${ }^{29}$ Ji-Hai Xu, Y. Ren, and C.S. Ting, Phys. Rev. B 52, 7663 (1995).

${ }^{30}$ M. Fogelström, D. Rainer, and J.A. Sauls, Phys. Rev. Lett. 79, 281 (1997)

${ }^{31}$ T. Löfwander, V.S. Shumeiko, and G. Wendin, Phys. Rev. B 62, R14 653 (2000).

${ }^{32}$ A.I.M. Rae, Phys. Rev. Lett. 84, 2235 (2000).

${ }^{33}$ R. Haslinger and R. Joynt, J. Phys.: Condens. Matter 12, 8179 (2000).

${ }^{34}$ A.A. Golubov and M.Yu. Kupriyanov, JETP Lett. 67, 501 (1998).

${ }^{35}$ Guo-meng Zhao, Phys. Rev. B 64, 024503 (2001).

${ }^{36}$ H.C. Lee and Han-Yong Choi, Phys. Rev. B 65, 174530 (2002).

${ }^{37}$ G. Eilenberger, Z. Phys. 214, 195 (1968).

${ }^{38}$ Yu.N. Ovchinnikov, Sov. Phys. JETP 29, 853 (1969).

${ }^{39}$ J.W. Serene and D. Rainer, Phys. Rep. 101, 221 (1983).

${ }^{40}$ G.B. Arnold and R.A. Klemm, Phys. Rev. B 62, 661 (2000).

${ }^{41}$ Vladioni Z. Kresin and Stuart A. Wolf, Phys. Rev. B 41, 4278 (1990).

${ }^{42}$ Yu.V. Sharvin, Sov. Phys. JETP 21, 655 (1965).

${ }^{43}$ F. J. Culetto, G. Kieselmann, and D. Rainer, in 17th International Conference on Low Temperature Physics, LT-17, edited by U. Eckern, A. Schmid, W. Weber, and H. Wühl (North-Holland, Amsterdam, 1984), p. 1027.

${ }^{44} \mathrm{G}$. Lüders and K.D. Usadel, in The Method of the Correlation Function in Superconductivity Theory, Springer Tracts in Modern Physics, Vol. 56, edited by G. Höhler (Springer, Berlin, 1971), p. 133, Eq. (15.42).

${ }^{45}$ The contribution of the zero-energy state is implicitly taken into account in the quasiclassical theory as can be seen from the sign change in the critical current at low temperature. See Weiyi Zhang and Zidan Wang, Phys. Rev. B 65, 144527 (2002). 